\title{
Diagnosis and management of toxic shock syndrome after breast reconstructive procedures with silicone implants
}

\author{
Minseo Kim, Inhoe Ku, Ung Sik Jin \\ Department of Plastic and Reconstructive Surgery, Seoul National University Hospital, Seoul, Korea
}

\begin{abstract}
Breast implant insertion is one of the most commonly used methods for breast reconstruction after total mastectomy. However, infection is a common postoperative complication of implant insertion. In most cases, these infections can be managed with antibiotics and supportive therapy. However, severe septic conditions, such as toxic shock syndrome (TSS), can sometimes occur. TSS is an extremely rare but life-threatening complication, for which early diagnosis and proper management play a crucial role in determining patients' outcome. Although only 16 cases of TSS after breast implant insertion have been reported in the literature, most of those cases involved a serious clinical course. The reason for the seriousness of the clinical course of TSS in these cases is that the initial impression and presentation of these patients are nonspecific, and patients can easily be misdiagnosed as having a simple upper respiratory infection, causing the underlying condition to be neglected. Herein, we present two patients who were diagnosed with TSS after receiving breast reconstruction surgery via a silicone implant after total mastectomy. Both patients were misdiagnosed at the initial examination since they showed no local infectious signs on the postoperative wound.
\end{abstract}

Keywords Breast / Reconstructive surgery / Implant / Toxic shock syndrome

\author{
Correspondence: Ung Sik Jin \\ Department of Plastic and \\ Reconstructive Surgery, Seoul \\ National University Hospital, 101 \\ Daehak-ro, Jongro-gu, Seoul 03080, \\ Korea \\ Tel: +82-2-2072-2375 \\ Fax: +82-2-741-3985 \\ E-mail: usj1011@snu.ac.kr
}

\section{INTRODUCTION}

The clinical manifestation of toxic shock syndrome (TSS) includes multiple symptoms and signs such as fever, rash, desquamation of the extremities, hypotension, and multiple organ failure, which can lead to serious outcomes [1]. Since TSS is extremely rare and its presentation changes throughout the clinical course, most plastic surgeons do not consider it as an initial diagnostic possibility when patients present with the corresponding symptoms. Therefore, we present two cases at our institu- tion to share our experiences and to aid in the early diagnosis and treatment of this condition.

\section{CASES}

\section{Case 1}

A 31-year-old woman presented to the clinic with a complaint of left breast deformity after total mastectomy owing to a breast cancer diagnosis 3 years ago. The patient had undergone delayed breast reconstruction with a pedicled latissimus dorsi flap 
with tissue expander insertion (Natrelle 133 FV, Allergan, Dublin, Ireland; $300 \mathrm{cc}$ ). Nine months later, the tissue expander was removed and a silicone implant (BellaGel anatomical textured, Hansbiomed Inc., Seoul, Korea; moderate height, medium projection; $260 \mathrm{cc}$ ) was inserted, along with breast augmentation surgery (BellaGel round micro-textured; medium projection; $200 \mathrm{cc}$ ) for the contralateral breast. After the last operation, the patient was discharged without any postoperative complications on postoperative day (POD) 4. The negative-pressure drainage was removed on POD 16 at an outpatient clinic follow-up. Later on the same day, the patient was admitted to the emergency department with a high fever (up to $40.3^{\circ} \mathrm{C}$ ); however, she showed no symptoms or signs of local infection such as redness or tenderness. The patient was diagnosed with an upper respiratory infection and was discharged.

On POD 18, the patient was urgently admitted to the emergency department with a high fever $\left(40.3^{\circ} \mathrm{C}\right)$ and low blood pressure (systolic blood pressure [SBP], $83 \mathrm{mmHg}$; diastolic blood pressure [DBP], $46 \mathrm{mmHg}$ ). The patient also complained of nausea and vomiting, along with chills and myalgia. Diffuse macular erythroderma was observed on the patient's trunk, bilateral arms, and bilateral legs. The initial lab results of the patient showed a white blood cell (WBC) count of $6.57 \times 10^{3} / \mu \mathrm{L}$, a C-reactive protein (CRP) level of $29.42 \mathrm{mg} / \mathrm{dL}$, elevated aspartate transaminase and alanine transaminase levels $(123 \mathrm{IU} / \mathrm{L}$ and $92 \mathrm{IU} / \mathrm{L})$, and a low platelet count $\left(77 \times 10^{3} / \mu \mathrm{L}\right)$. Infection of the surgical site was initially suspected, but an examination of the operative sites showed no recognizable inflammatory signs suggesting infection (Fig. 1), except for slight swelling. Contrastenhanced chest computed tomography merely suggested a mild complex fluid collection in her left breast (Fig. 2).

Prophylactic antibiotic treatment (intravenous [IV] vancomy-

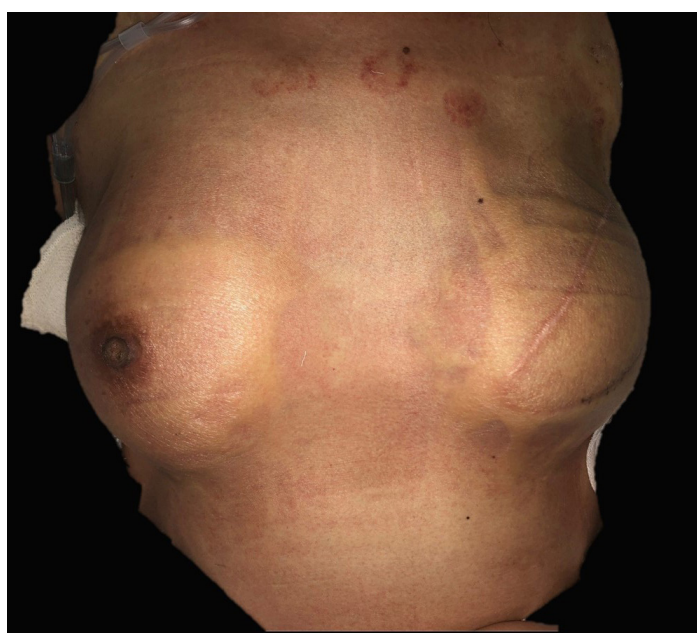

Fig. 1. Medical photograph of the patient's preoperative condition. cin, $1 \mathrm{~g}$ twice a day) was started immediately. Immediate wound exploration the day after admission was done under general anesthesia with thorough irrigation with an antibiotic solution and implant change procedures in the bilateral breasts. The intraoperatively examined wound showed minimal clear fluid collection, rather than infectious turbid discharge or necrotic tissue. Nevertheless, to rule out infection, tissue cultures from both breasts were performed.

After immediate wound exploration and irrigation, the general status of patient stabilized, with an SBP of $97 \mathrm{mmHg}$, DBP of 65 $\mathrm{mmHg}$, and body temperature of $37.1^{\circ} \mathrm{C}$ on POD 2. The surgical site of the patient did not show any signs of local infection on POD 3 (Fig. 3). On POD 5, tissue culture from the left breast wound grew methicillin-resistant Staphylococcus aureus (MRSA),

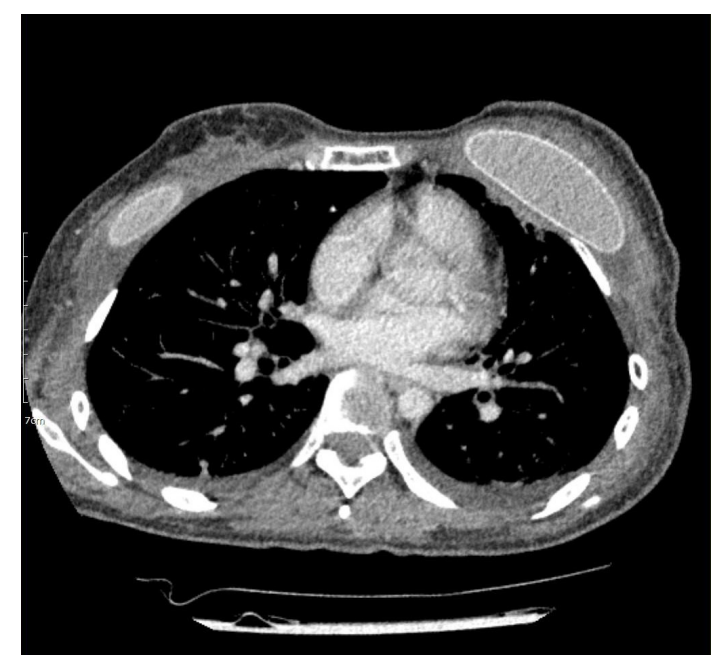

Fig. 2. Topographic imaging of the patient. Preoperative chest computed tomography on postoperative day 18 , showing mild complex fluid collection.

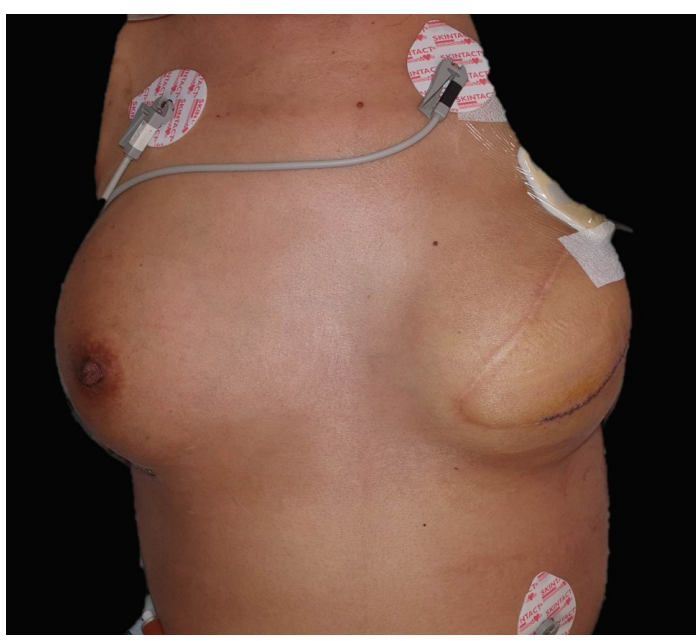

Fig. 3. Photograph of patient on postoperative day 3. 


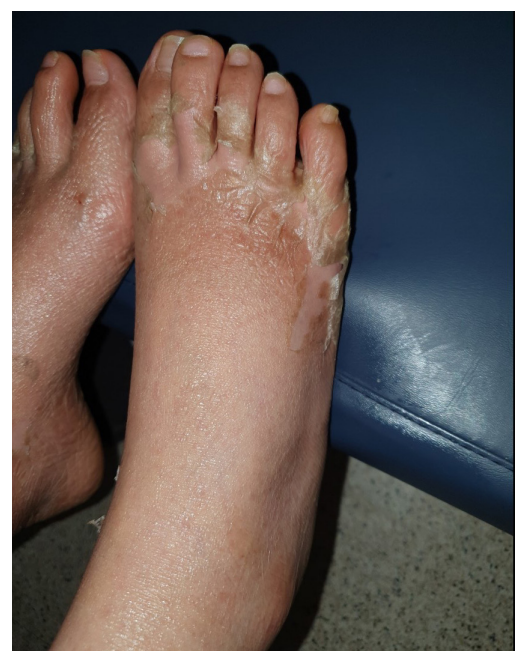

Fig. 4. Clinical photograph of the patient. Desquamation of bilateral feet as the natural course of toxic shock syndrome.
Table 1. Diagnostic criteria of toxic shock syndrome

\begin{tabular}{l}
\hline \multicolumn{1}{c}{ Criteria of toxic shock syndrome } \\
\hline 1. Fever $>38.9^{\circ} \mathrm{C}$ \\
2. Rash (diffuse macular erythroderma) \\
3. Desquamation (1-2 weeks after onset, particularly the palms and soles) \\
4. Hypotension \\
5. Multisystem organ involvement of 3 or more systems \\
a. Gastrointestinal (vomiting or diarrhea at illness onset) \\
b. Musculoskeletal (myalgia or elevated creatinine phosphokinase) \\
c. Mucous membrane (vaginal, oropharyngeal, or conjunctival hyperemia) \\
d. Renal \\
e. Hepatic (elevated liver function tests) \\
f. Hematologic (thrombocytopenia) \\
g. CNS (altered mental status) \\
6. Negative results to blood, throat, or CSF cultures \\
7. Positive culture for Staphylococcus aureus
\end{tabular}

CNS, central nervous system; CSF, cerebrospinal fluid.

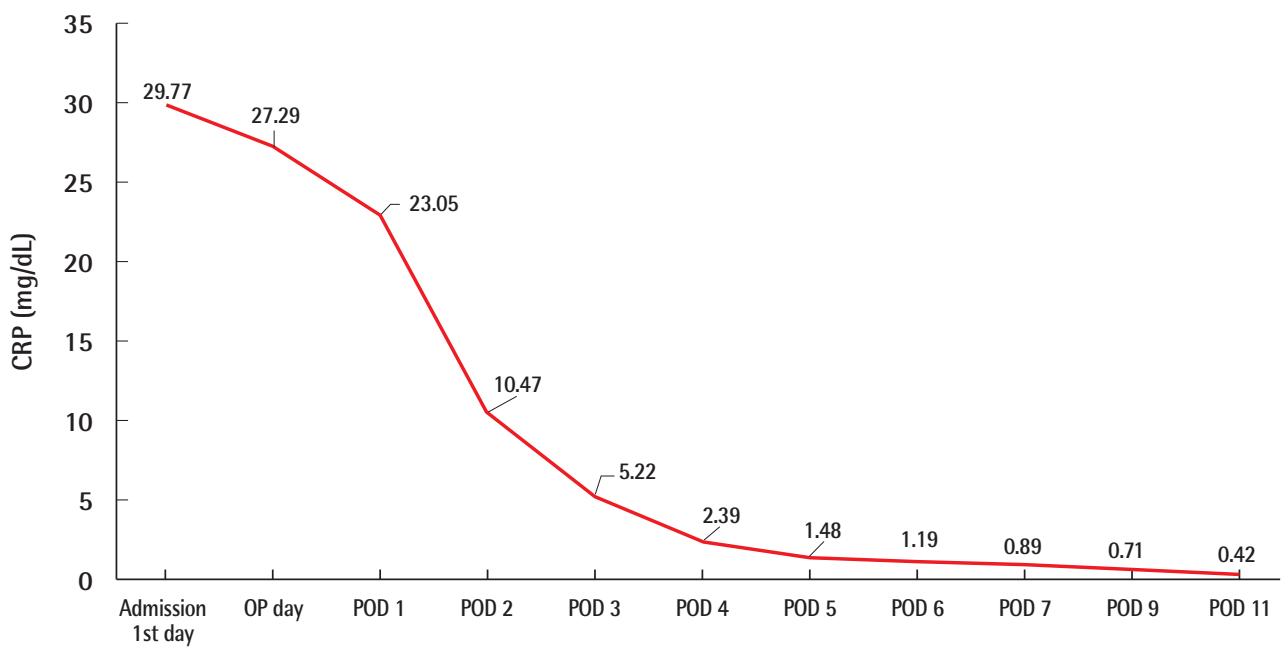

Fig. 5. Values of CRP of case 1. The levels of inflammatory markers such as CRP also decreased to normal within 7 days after admission. CRP, C-reactive protein; OP, operation; POD, postoperative day.

which is a causative microorganism of TSS. The antibiotics regimen was changed to IV vancomycin and clindamycin.

Desquamation of the hands and feet appeared on the sixth day after admission (Fig. 4). The levels of inflammatory markers such as CRP also decreased to normal within 7 days, and the patient was discharged on POD 11 (Fig. 5). To summarize, the patient showed fever, rash, desquamation, hypotension, and multisystem involvement (high hepatic enzyme levels, gastrointestinal symptoms, low platelet count), which fit the criteria of TSS as shown in Table 1.

\section{Case 2}

A 55-year-old patient underwent tissue expander removal (Natrelle $133 \mathrm{MV} ; 250 \mathrm{cc}$ ) and implant insertion (BellaGel anatom- ical textured; tall height, medium projection; $250 \mathrm{cc}$ ) due to breast cancer found 1 year previously. The patient was discharged without any complications on POD 2, and her vital signs were stable. On POD 5, the patient was admitted to the emergency department with a high fever of $39.9^{\circ} \mathrm{C}$, low blood pressure (SBP, $84 \mathrm{mmHg}$; DBP, $52 \mathrm{mmHg}$ ) and respiratory difficulty. The initial lab results showed an elevated WBC count of $11.04 \times$ $10^{3} / \mu \mathrm{L}$, a platelet count of $203 \times 10^{3} / \mu \mathrm{L}$, and a high CRP level of $5.95 \mathrm{mg} / \mathrm{dL}$. The surgical site showed slight swelling, along with breast tenderness. The patient complained of vomiting, diarrhea, and myalgia. An emergent operation for wound irrigation and drain insertion was performed immediately on the day the patient was admitted, and IV vancomycin $(750 \mathrm{mg}$ three times a day) and meropenem ( $1 \mathrm{~g}$ three times a day) were ad- 


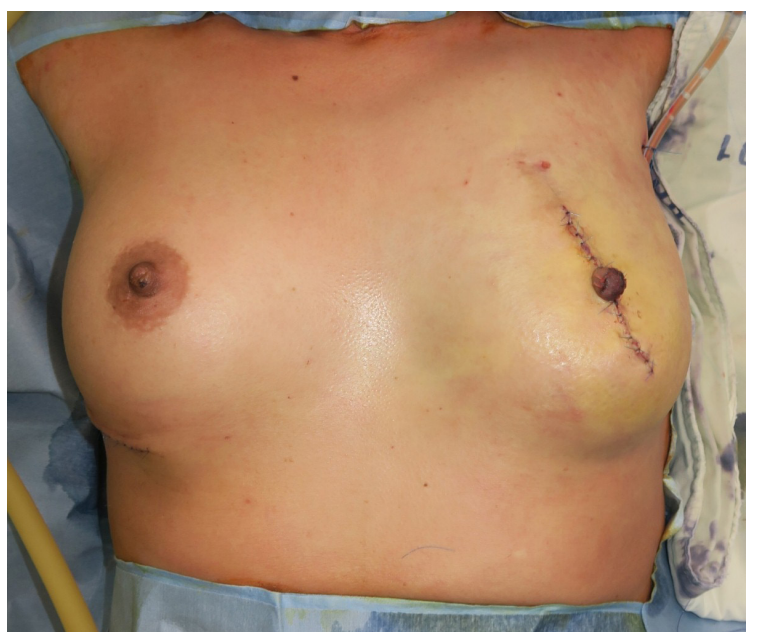

Fig. 6. Clinical photograph of surgical site. Postoperative medical photograph of the patient (wound irrigation and drain insertion).

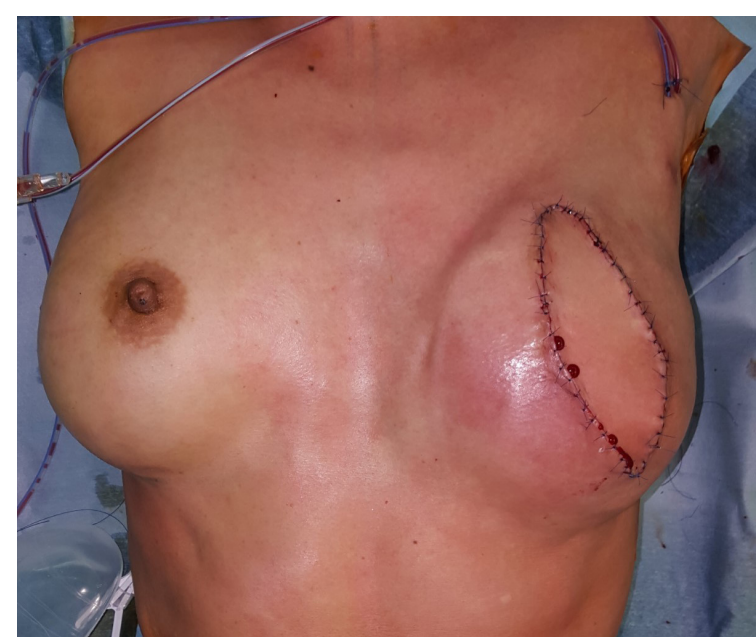

Fig. 8. Clinical photograph of surgical site. Postoperative medical photograph of the patient (reconstruction with a pedicled latissimus dorsi flap and implant change).

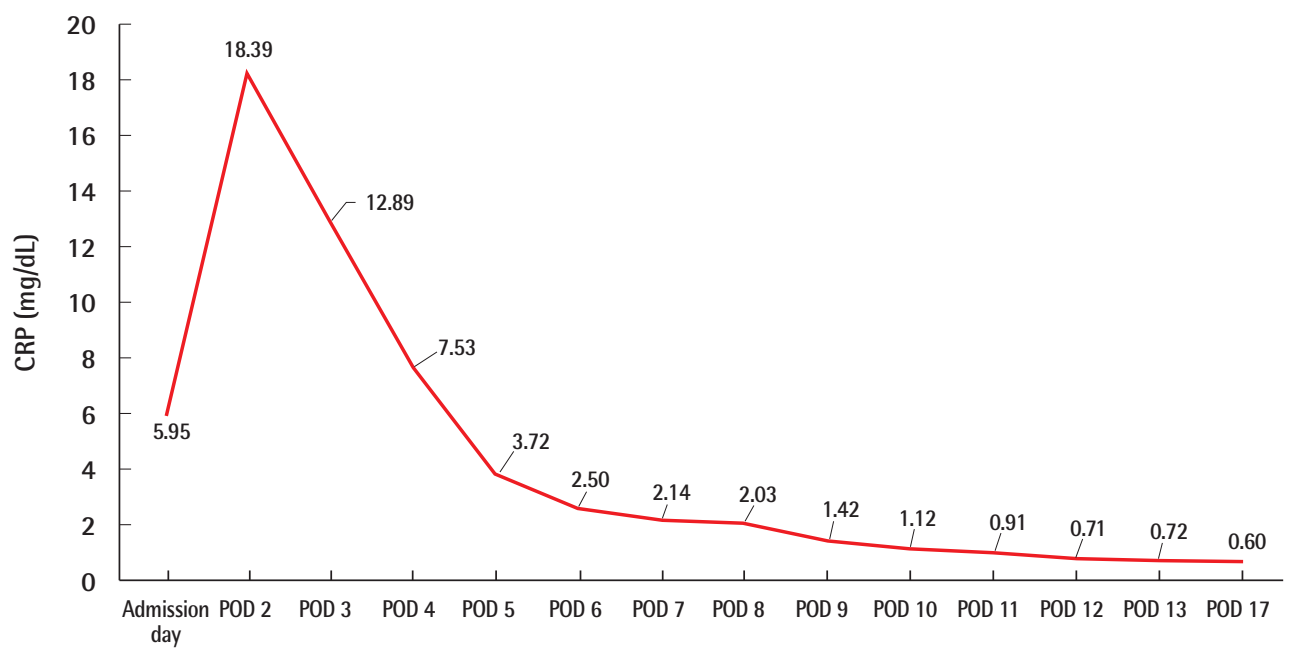

Fig. 7. Values of CRP of case 2. The levels of inflammatory markers such as CRP level also increased on POD 2, decreased afterwards, and stabilized. CRP, C-reactive protein; POD, postoperative day.

ministered (Fig. 6). Tissue culture from the breast wound grew MRSA. The WBC count, which was $11.04 \times 10^{3} / \mu \mathrm{L}$ on admission, increased to $19.59 \times 10^{3} / \mu \mathrm{L}$ on POD 1 and decreased afterwards. The platelet count, which was $203 \times 10^{3} / \mu \mathrm{L}$ on the day of admission, gradually decreased to $75 \times 10^{3} / \mu \mathrm{L}$ by POD 4 , increased afterwards, and then stabilized. The CRP level also increased to $18.39 \mathrm{mg} / \mathrm{dL}$ by POD 2, decreased afterwards, and stabilized (Fig. 7). However, mild fever persisted even after the operation, the wound site continued to show redness, and the patient complained of tenderness at the surgical site. Therefore, on POD 13, an implant change (BellaGel anatomical textured; tall height, medium projection, BATT-M; $220 \mathrm{cc}$ ) was performed and the patient was discharged 5 days later. The patient visited the outpatient clinic on a regular basis; however, the surgical site continued to show wound dehiscence and thin skin.
Breast reconstruction with a latissimus dorsi flap and implant change (BellaGel anatomical textured; tall height, medium projection, BATT-M; $220 \mathrm{cc}$ ) was performed on POD 60, and no subsequent complications occurred (Fig. 8). This patient also presented with fever, hypotension, and multisystem failure (gastrointestinal symptoms, muscular symptoms, low platelet count). Records of rash or desquamation were not found, but her general presentation fit the typical profile of TSS.

\section{DISCUSSION}

In these cases, the patients' initial vital signs and laboratory results suggested a postoperative infection, but the absence of focal signs of infection, elevated hepatic enzyme levels, complaints of gastroenterological symptoms, rash over the body, and low 
platelet count constituted an extraordinary course for a routine prosthetic infection. The authors suspected TSS since the initial physical examination and laboratory results were in accordance with the diagnosis criteria of TSS. Isolation of MRSA from tissue culture and desquamation of the hands and feet (in one of the patients) led to a conclusive diagnosis of TSS.

Early suspicion of TSS and proper management with surgical exploration of the wound are crucial in the diagnosis and treatment of TSS; thus, to achieve the best possible outcomes, clinicians should be attentive to the possibility of this disease even when there are no infection signs at the local wound area. Once TSS has been diagnosed, proper management is imperative. The main components of its management are resuscitation, removal of the source of infection, and a rational choice of antibiotics [2]. In most cases, patients' vital signs are unstable; therefore, clinicians should immediately perform resuscitation using fluid, respiratory, and if necessary inotropic support. Once the patient's medical condition is stabilized, the progression of TSS should be stopped by removing the source of infection and using proper antibiotics, since systemic inflammation is caused by the superantigen produced by bacteria. However, the early diagnosis of TSS has been a dilemma for clinicians due to its ambiguous symptoms and signs.

Unfortunately, definitive diagnostic tools for TSS have yet to be developed, and the diagnosis still depends on examination findings and the clinician's opinion. TSS can occur after even simple procedures such as skin removal. Even young healthy patients cannot be excluded from the diagnosis [3]. Therefore, surgeons should always keep TSS in mind, even for minimally invasive procedures. TSS can be ambiguous despite its potentially fatal outcomes, and once the syndrome is suspected, prompt surgical intervention and wound site culture are essential even if the surgical site does not clearly present signs of local infection [3].

Since definitive diagnostic procedures and management guidelines for TSS have not yet been developed, and the syndrome can be ambiguous, the timing and planning for management can be confusing for inexperienced surgeons. Therefore, further studies are required.

\section{NOTES}

\section{Conflict of interest}

No potential conflict of interest relevant to this article was reported.

\section{Ethical approval}

The study was approved by the Institutional Review Board of Seoul National University Hospital (IRB No. 2007-200-1144) and performed in accordance with the principles of the Declaration of Helsinki. Written informed consent was obtained.

\section{Patient consent}

The patients provided written informed consent for the publication and the use of their images.

\section{Author contribution}

Conceptualization and data curation: M Kim, I Ku, US Jin. Formal analysis: US Jin. Methodology, project administration, and visualization: M Kim, I Ku, US Jin. Writing - original draft: M Kim, I Ku, US Jin. Writing - review \& editing: M Kim, I Ku, US Jin.

\section{ORCID}

Minseo Kim https://orcid.org/0000-0002-5536-3920

Inhoe $\mathrm{Ku} \quad \mathrm{https} / /$ orcid.org/0000-0002-3046-6800

Ung Sik Jin https://orcid.org/0000-0001-5096-6207

\section{REFERENCES}

1. Stevens DL, Bisno AL, Chambers HF, et al. Practice guidelines for the diagnosis and management of skin and soft tissue infections: 2014 update by the Infectious Diseases Society of America. Clin Infect Dis 2014;59:e10-52.

2. Wilkins AL, Steer AC, Smeesters PR, et al. Toxic shock syndrome: the seven Rs of management and treatment. J Infect 2017;74 Suppl 1:S147-52.

3. Celie KB, Colen DL, Kovach SJ 3rd. Toxic shock syndrome after surgery: case presentation and systematic review of the literature. Plast Reconstr Surg Glob Open 2020;8:e2499. 